\title{
Treatment of an abscess after massive autologous fat transplantation for breast augmentation with real-time ultrasonography-guided liposuction
}

\author{
Seo Gil Choi, Junekyu Kim \\ Department of Plastic and Reconstructive \\ Surgery, Kangbuk Samsung Hospital, \\ Sungkyunkwan University School of \\ Medicine, Seoul, Korea
}

Many studies have demonstrated that an autologous fat graft (AFG) can be a successful alternative to prosthetic breast augmentation; however, with the increasing use of this method, there are also increasing reports of complications. We report a case of severe infection after augmentation mammoplasty with a massive AFG. A 23-year-old woman had undergone primary AFG for breast augmentation. The remaining fat was reused for secondary graft 46 days after the primary transplantation. The patient was referred to our hospital with infectious signs on both breasts. Antibiotics were administered according to the bacterial culture result of the abscess aspirated with ultrasonography (US) guidance. During antibiotic treatment, two US-guided abscess aspirations were performed. Although follow-up magnetic resonance imaging demonstrated reduced abscess and fluid collection after aspiration, the skin of the lower pole of the right breast was perforated. We performed liposuction with real-time US guidance for abscess localization. The abscess and necrotic fat tissue were removed through liposuction. Thereafter, the symptoms, signs, and laboratory and radiologic findings demonstrated complete improvement. The patient was successfully treated while maintaining the breast contour through localized abscess removal, with a much smaller liposuction scar than would have resulted from aggressive excision or drainage.

Keywords Mammaplasty / Infection / Liposuction
This article was presented at the 7th Research and Reconstruction Forum on April 20-21, 2017, in Daejeon, Korea. hibited because it could interfere with the detection of breast cancer, several studies have reported little impact of fat transplantation on cancer detection $[2,3]$. In light of the results of previous studies about safety with respect to cancer detection and improvements of the technique, autologous fat transplantation for breast reconstruction and breast augmentation has attracted much attention, and has been performed by many plastic surgeons and by many physicians not board-certified as plastic surgeons.

However, even with sophisticated and innovative techniques, autologous fat transplantation is still a challenging procedure with unpredictable outcomes in terms of graft viability and fat necrosis. Non-absorbed fat necrosis may lead to complications such as an oily cyst, sclerotic induration, calcified solid tumors, and infections [4]. In a large-scale study, only one local infection was reported in 880 fat graft procedures in the breast, and it was treated without surgical intervention [5]. Nonetheless, cases of severe infections and aggressive surgical treatment have been also reported [6]. We 
report a case of a patient presenting with multiple abscesses induced by fat necrosis after massive autologous transplantation for breast augmentation, to share an effective treatment plan for such complications and to review the appropriate measures to prevent complications.

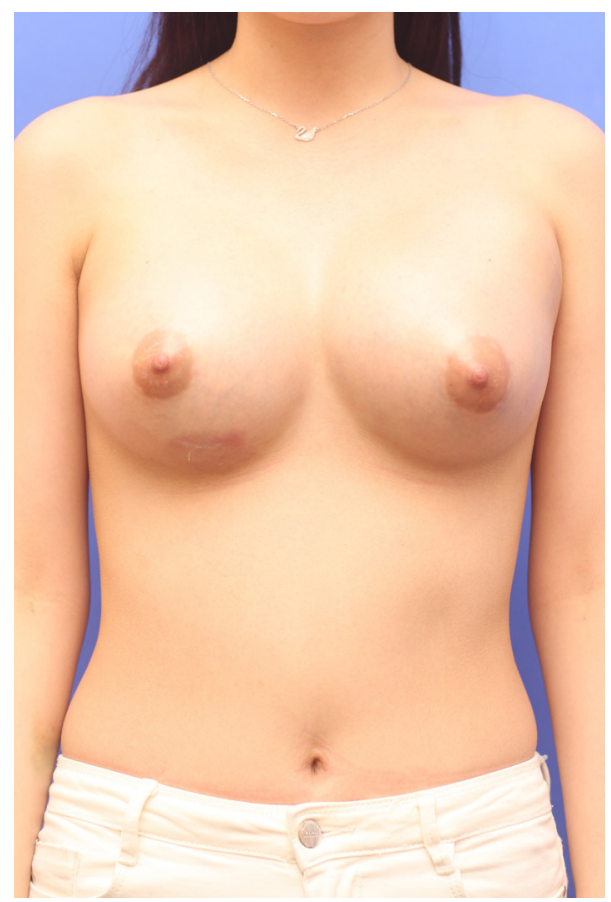

Fig. 1. The patient presented with pain and tenderness in both breasts. The skin of the lower pole of the right breast was thinned.

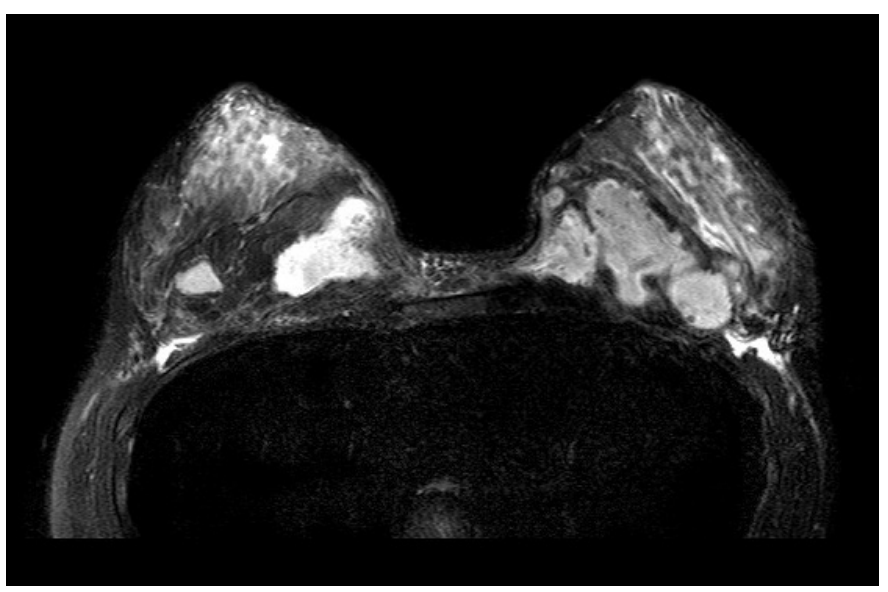

Fig. 2. There were multiple abscess pockets in the subpectoral and retromammary areas. Moreover, the abscess pocket in the right breast was almost in contact with the skin and was just about to penetrate the skin.

\section{CASE REPORT}

A 23-year-old female patient had undergone autologous transplantation with fat grafted from both thighs ( $150 \mathrm{~mL}$ per breast). Much more than $300 \mathrm{~mL}$ of fat had been harvested, and the remaining fat was reused for a secondary graft procedure ( $110 \mathrm{~mL}$ per breast) 46 days after the primary transplantation. The remaining fat had been stored in a freezer that was not for medical use.

Starting on the 2 nd day after the operation, the patient started to show firmness, pain, and tenderness in her right breast. Therefore, she revisited the clinic where she had undergone surgery and was prescribed with cephalosporin and vancomycin antibiotics for about 2 weeks. However, the symptoms worsened. When she was referred to Kangbuk Samsung Hospital, the skin of the lower pole of the right breast was thin and there were symptoms of pain, tenderness, and redness in both breasts (Fig. 1).

Immediately after the patient's admission, we began to adminis-
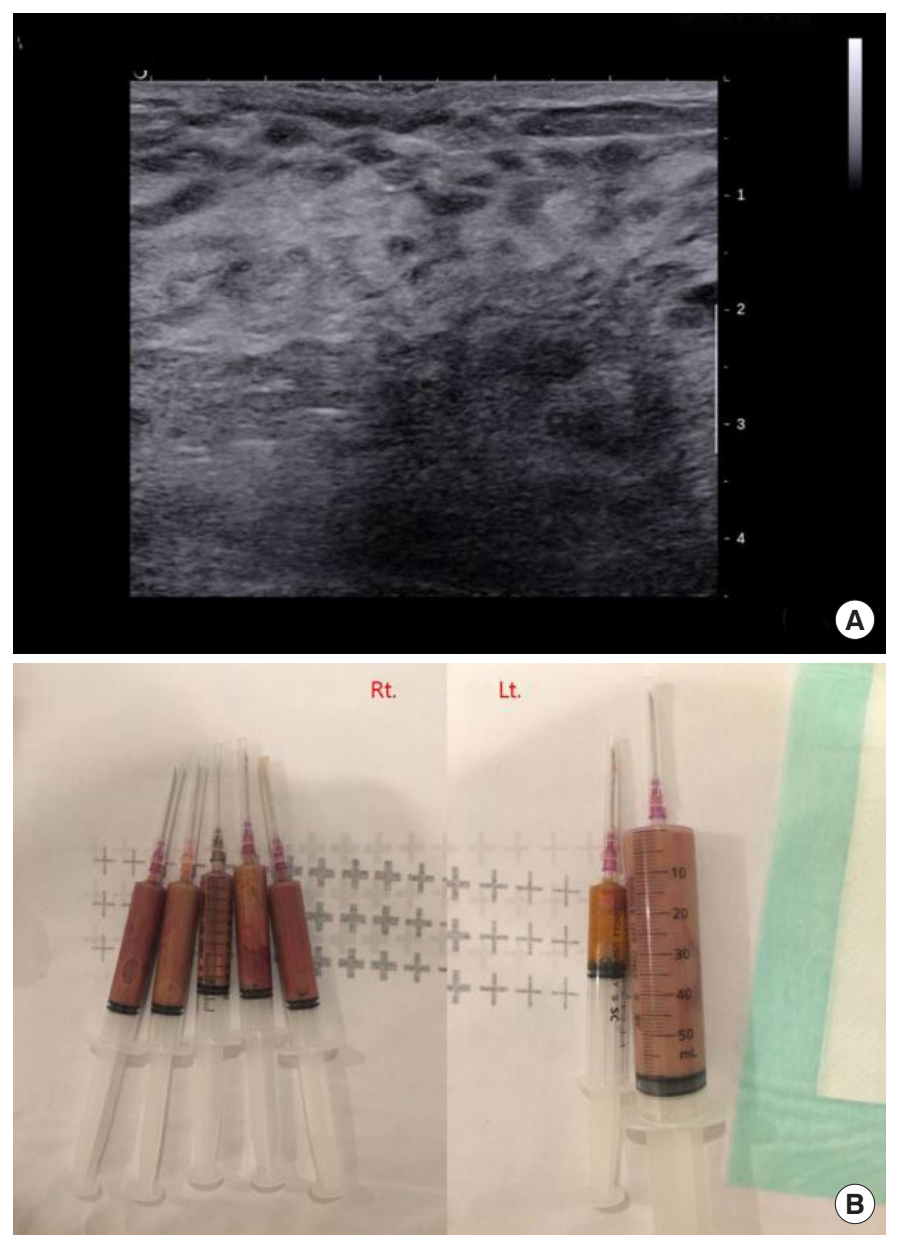

Fig. 3. (A) Initial breast ultrasonography (US): breast US revealed multiple loculated hyperechoic fluid collections in both breasts. (B) Secondary aspiration: the abscess content was removed $(70 \mathrm{~mL}$ from the right breast and $65 \mathrm{~mL}$ from the left breast) through US-guided aspiration. Rt, right; Lt, left. 
ter empirical antibiotics (cefepime, a fourth-generation cephalosporin) in cooperation with the department of infectious diseases. The initial magnetic resonance imaging (MRI) revealed fluid collection in the subpectoral area and parenchyma that contained fat under the muscles of both breasts. This was thought to correspond to multiple abscesses due to the infected autologous fat graft. The skin of the right lower pole was almost in contact with the fluid collection, which was just about to penetrate the skin (Fig. 2).

On the 2nd day of hospitalization, ultrasonography (US)-guided

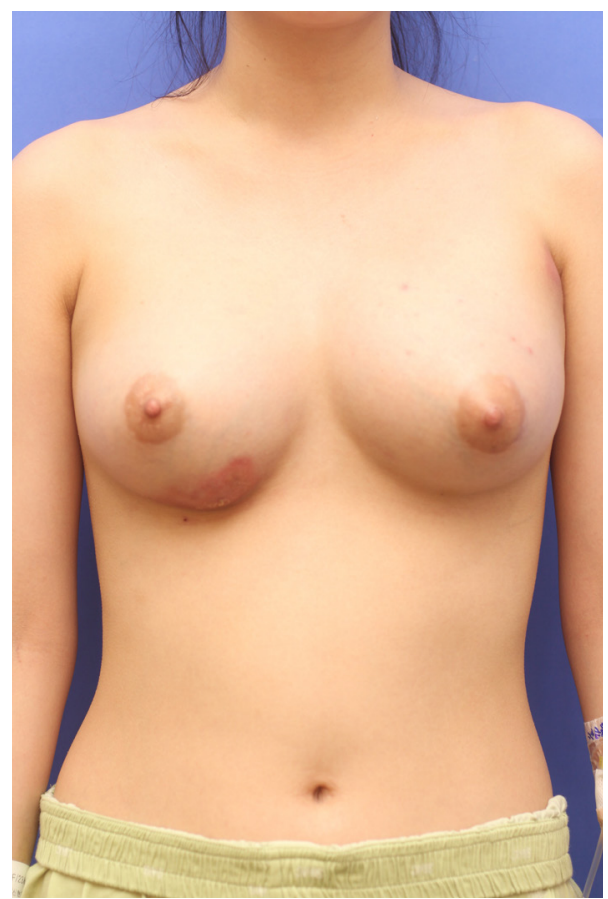

Fig. 4. On hospital day 14, a skin fistula on the lower pole of the right breast and newly developed redness on the left pre-axillary area were observed.

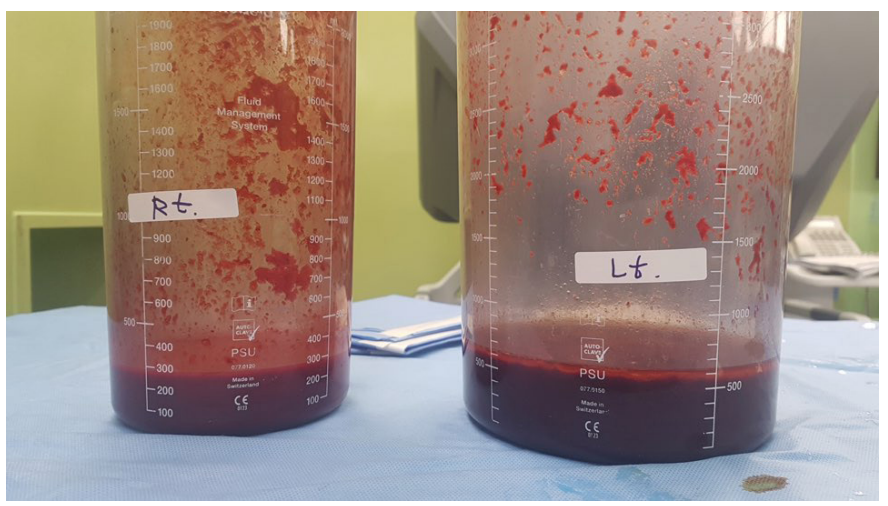

Fig. 5. Abscess and necrotic fat tissue with bloody discharge was removed using liposuction equipment (power-assisted liposuction) with real-time ultrasonography guidance in the operating room $1300 \mathrm{~mL}$ from the right breast, $650 \mathrm{~mL}$ from the left breast). Rt, right; Lt, left. aspiration was performed in cooperation with a radiologist to remove the pus $(23 \mathrm{~mL}$ from the right breast and $35 \mathrm{~mL}$ from the left breast). Methicillin-resistant Staphylococcus aureus (MRSA) and Klebsiella pneumoniae were identified from the bacterial cultures of the aspirated abscess. Therefore, the antibiotics were changed to vancomycin and ceftriaxone. Follow-up MRI performed 1 week after antibiotic treatment showed a slight decrease in the size of the abscess pocket, although multiple abscesses remained and there was more liquefaction. Thus, we decided to perform secondary USguided aspiration and removed 70 and $65 \mathrm{~mL}$ of abscess content from the right and left breasts, respectively (Fig. 3).

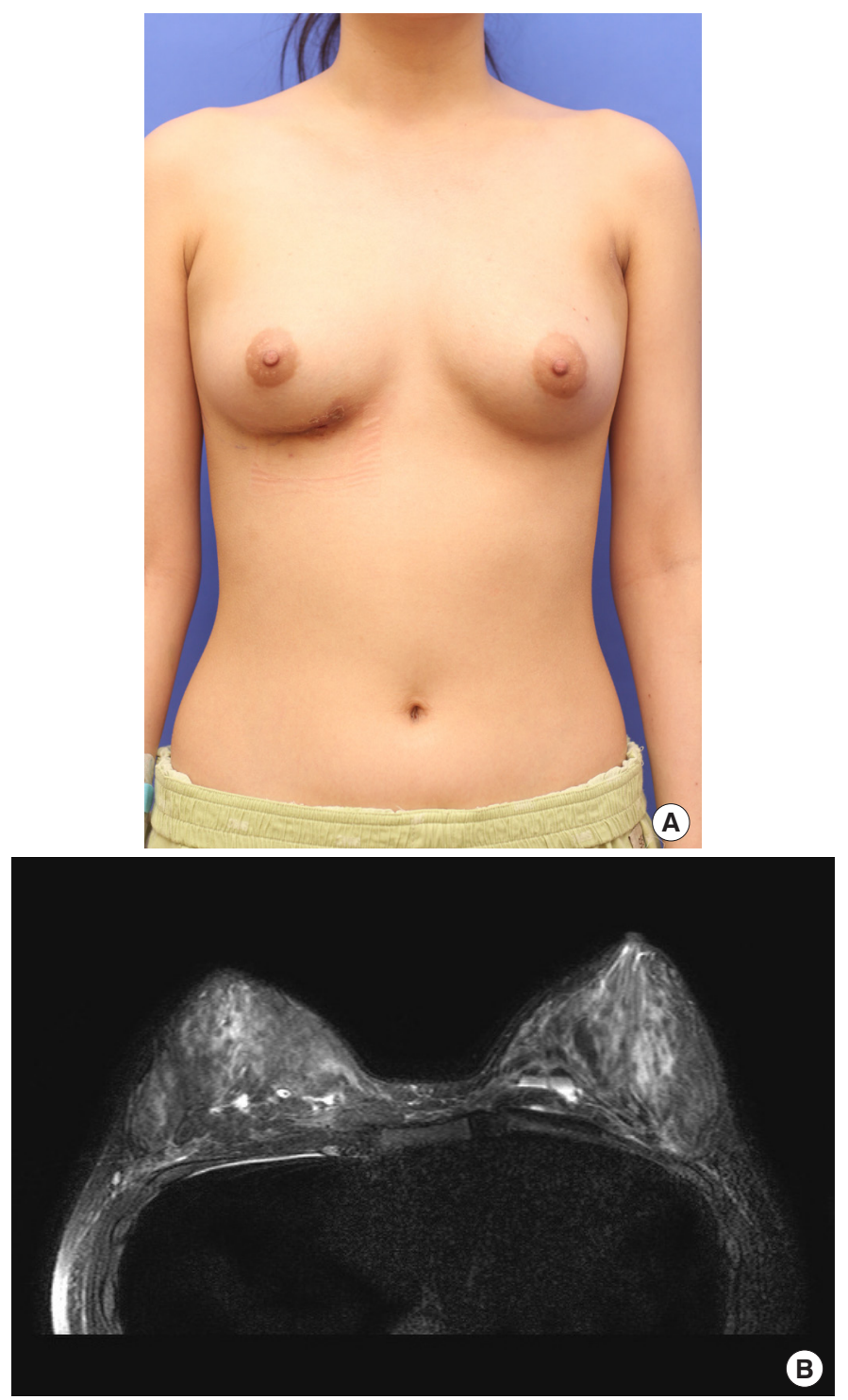

Fig. 6. (A) The local infection signs subsided and no more purulent discharge was observed, while the natural and aesthetically favorable contour of both breasts was preserved with a minimal wound. (B) A markedly reduced abscess pocket was observed on follow-up magnetic resonance imaging. 
The symptoms showed repeated cycles of improvement and worsening. Meanwhile, a skin fistula occurred in the lower pole of the right breast and newly developed redness was observed in the left axillary area (Fig. 4). Therefore, we decided to remove the residual abscess through liposuction under general anesthesia, with real-time US guidance utilizing an iU22 device (Philips Healthcare, Bothell, WA, USA). Images were acquired using a combination of L12-5 and L9-3 probes. The incision for liposuction involved minimal extension of the skin fistula on the lower pole of the right breast and a stab incision measuring roughly $1 \mathrm{~cm}$ on the left axilla. Realtime US guidance enabled us to localize the abscess pocket and distinguish it from normal healthy fat tissue. Thereby, we were able to remove necrotic fat tissue, the abscess content, infected tissue, and bloody discharge while preserving as much normal adipose tissue as possible ( $300 \mathrm{~mL}$ from the right breast and $650 \mathrm{~mL}$ from the left breast) (Fig. 5). A natural drainage tube was inserted into the right breast, and a closed suction drainage was inserted into the left breast. On the third day after liposuction, the drainage was removed and the incision wounds were closed. On postoperative day 7, the laboratory findings of infection and inflammation had normalized. Further, the symptoms and signs of local infection (pain, tenderness, redness, purulent discharge, and swelling) subsided, and follow-up MRI revealed a markedly improved state of the abscess in both breasts (Fig. 6).

\section{DISCUSSION}

After Coleman introduced the structural graft, autologous fat grafting has been suggested as a possible alternative to implant surgery for breast enlargement or breast reconstruction, and its effects and safety have been studied by several authors $[7,8]$. However, the details of procedures and methods of harvesting, storage, and transplantation of fat have been controversial and have been conducted in idiosyncratic ways by different surgeons based on anecdotal evidence. As a result, the reported graft survival rates and complications have been variable.

Complications after autologous fat transplantation include unfavorable scarring, fat necrosis, infection, and inadequate correction $[4,5]$. Complications that usually occur in the short term include edema, hematoma, infection, granuloma and cyst formation, and resorption. Fat necrosis is characterized by oily cysts, sclerotic induration, and calcified solid tumors. These non-absorbed areas of fat are vulnerable to infection because of a lack of vascularization. However, the definitive cause of infection after massive autologous fat transplantation has not been revealed by large-scale studies. In previous studies and case reports, Escherichia coli infection due to skin contamination was reported to be the most common cause; however, MRSA and K. pneumoniae were detected in the abscess culture of the present patient [9]. A prospective study in Australia found that $60 \%$ of all soft tissue infections were caused by commu- nity-acquired MRSA [10]. In this patient, the mechanism causing the infection was unclear, but we assumed that infection occurred in the fat tissue because of improper storage.

As autologous fat transplantation has been widely used in the field of aesthetic plastic surgery and reconstruction, various studies have been conducted on the method of cryopreservation for harvested fat. However, there is also no widely accepted method of freezing and thawing harvested fat. Shoshani et al. [11] reported that rapid freezing at $-20^{\circ} \mathrm{C}$ without a cooling process or a cryopreservation agent is the safest and most optimal method to preserve the viability of fat, whereas another study observed no differences in freezing at $-20^{\circ} \mathrm{C},-80^{\circ} \mathrm{C}$, and $-196^{\circ} \mathrm{C}$ [12]. A recent study concluded that controlled slow cooling $\left(1-2^{\circ} \mathrm{C} / \mathrm{min}\right.$ from $22^{\circ} \mathrm{C}$ to $30^{\circ} \mathrm{C}$ ) followed by transfer into liquid nitrogen, and then fast thawing by stirring in a $37^{\circ} \mathrm{C}$ water bath until the fat is thoroughly thawed, is the best method with respect to fat viability and safety [13]. Although it is important that the harvested fat is frozen, thawed, and reused using a proper and safe procedure, in this case, the fat was stored in a normal freezer (not for medical use). It can be assumed that the inappropriate storage of the reused fat was the cause of infection, because the symptoms appeared immediately after the secondary fat transplantation.

Once infection has occurred in transplanted fat, it is important to remove infected and necrotic tissue as soon as possible. Therefore, aggressive incision and drainage or excision are recommended. However, because the patient was a young woman who worked in the entertainment industry, it was also important to minimize scarring and to maintain the breast contour. Therefore, after consultation with the patient, we first attempted fine-needle aspiration with antibiotic therapy. US confirmed the abscess pocket, and we attempted to reduce the pocket size through two rounds of aspiration. The remaining abscess and necrotic fat were also localized and removed using liposuction equipment with real-time US guidance in the operating room, preserving the normal adipose tissue as much as possible and maintaining the contour of the breast. Unlike invasive incision and drainage, which were reported in previous cases, the use of liposuction through a minimal incision allowed us to minimize the visible scar $[6,14]$. The incision on the right breast was made by extending the fistula in the lower pole by $1 \mathrm{~cm}$, and the left breast was incised by $1.5 \mathrm{~cm}$ at the axilla to hide the scar as close as possible to the abscess pocket that was located using US. Immediate and invasive incision and drainage should be performed if a life-threatening infection progresses. Otherwise, a progressive treatment plan using real-time US, as in this case, can provide more aesthetically favorable outcomes. Breast augmentation or reconstruction through autologous fat transplantation is often performed due to its advantages, which include a natural contour and texture and reduced prosthesis-associated complications. However, appropriate prevention and treatment procedures for the abovementioned complications should precede surgery [5]. 


\section{NOTES}

\section{Conflict of interest}

No potential conflict of interest relevant to this article was reported.

\section{Ethical approval}

The study was performed in accordance with the principles of the Declaration of Helsinki.

\section{Patient consent}

The patient provided written informed consent for the publication and the use of her images.

\section{ORCID}

Seo Gil Choi https://orcid.org/0000-0002-8945-2278

Junekyu Kim https://orcid.org/0000-0002-8904-5605

\section{REFERENCES}

1. Czerny A. Plastischer Ersatz der BrustdrosedurcheinLipoma. Chir Kongr Verhandl 1895;216:216-7.

2. Coleman SR, Saboeiro AP. Fat grafting to the breast revisited: safety and efficacy. Plast Reconstr Surg 2007;119:775-85.

3. Illouz YG, Sterodimas A. Autologous fat transplantation to the breast: a personal technique with 25 years of experience. Aesthetic Plast Surg 2009;33:706-15.

4. Lin JY, Song P, Pu LLQ. Management of fat necrosis after autologous fat transplantation for breast augmentation. Plast Reconstr Surg 2018; 142:665e-673e.
5. Delay E, Garson S, Tousson G, et al. Fat injection to the breast: technique, results, and indications based on 880 procedures over 10 years. Aesthet Surg J 2009;29:360-76.

6. Valdatta L, Thione A, Buoro M, et al. A case of life-threatening sepsis after breast augmentation by fat injection. Aesthetic Plast Surg 2001; 25:347-9.

7. Coleman SR. Facial recontouring with lipostructure. Clin Plast Surg 1997;24:347-67.

8. Coleman SR, Saboeiro AP. Primary breast augmentation with fat grafting. Clin Plast Surg 2015;42:301-6.

9. Moet GJ, Jones RN, Biedenbach DJ, et al. Contemporary causes of skin and soft tissue infections in North America, Latin America, and Europe: report from the SENTRY Antimicrobial Surveillance Program (1998-2004). Diagn Microbiol Infect Dis 2007;57:7-13.

10. Macmorran E, Harch S, Athan E, et al. The rise of methicillin resistant Staphylococcus aureus: now the dominant cause of skin and soft tissue infection in Central Australia. Epidemiol Infect 2017;145:2817-26.

11. Shoshani O, Ullmann Y, Shupak A, et al. The role of frozen storage in preserving adipose tissue obtained by suction-assisted lipectomy for repeated fat injection procedures. Dermatol Surg 2001;27:645-7.

12. Li BW, Liao WC, Wu SH, et al. Cryopreservation of fat tissue and application in autologous fat graft: in vitro and in vivo study. Aesthetic Plast Surg 2012;36:714-22.

13. Shu Z, Gao D, Pu LL. Update on cryopreservation of adipose tissue and adipose-derived stem cells. Clin Plast Surg 2015;42:209-18.

14. Lee KS, Seo SJ, Park MC, et al. Sepsis with multiple abscesses after massive autologous fat grafting for augmentation mammoplasty: a case report. Aesthetic Plast Surg 2011;35:641-5. 